\title{
On the molecular genetics of flexibility: The case of task-switching, inhibitory control and genetic variants
}

\author{
Sebastian Markett $\cdot$ Christian Montag • \\ Nora T. Walter • Thomas Plieger • Martin Reuter
}

Published online: 13 October 2011

(C) Psychonomic Society, Inc. 2011

\begin{abstract}
The adjustment of behavior to changing goals and environmental constraints requires the flexible switching between different task sets. Cognitive flexibility is an endophenotype of executive functioning and is highly heritable, as indicated by twin studies. Individual differences in global flexibility as assessed by reaction-time measurement in a task-switching paradigm were recently related to a single nucleotide polymorphism in the vicinity of the dopamine $\mathrm{d} 2$ receptor gene DRD2. In the present study, we assessed whether the DRD2 gene is related to backward inhibition, a control mechanism that contributes to cognitive flexibility by reducing proactive interference by no longer relevant task sets. We found that carriers of the DRD2 A1+ variant who have a lower striatal dopamine $\mathrm{d} 2$ receptor density than $\mathrm{A} 1$ - carriers show a larger backward inhibition effect. This is in line with previous results demonstrating increased behavioral flexibility in carriers of this genetic variant. The discussion relates the present finding to those of previous studies assessing the neurogenetic foundations of inhibitory control.
\end{abstract}

Keywords Cognitive control - Dopamine .

Prefrontal cortex $\cdot$ Pharmacology

Electronic supplementary material The online version of this article (doi:10.3758/s13415-011-0058-6) contains supplementary material, which is available to authorized users.

S. Markett $\cdot$ C. Montag $\cdot$ N. T. Walter $\cdot$ T. Plieger $\cdot$ M. Reuter

University of Bonn,

Bonn, Germany

S. Markett $(\bowtie)$

Institut fuer Psychologie,

Kaiser-Karl-Ring 9,

53111 Bonn, Germany

e-mail: sebastian.markett@uni-bonn-diff.de
Imagine your daily work in the office, a weekend off with your family, or a decent party night out with your friends. No matter which situation you are at you will probably engage in a wide range of different activities. Occasionally, you will need to disengage in a certain activity to start another one in order to meet up with your intentions or with constraints inflicted on you by the environment. Usually, goal-directed switching between different activities or tasks is found to be easy, but in the experimental laboratory, performance costs associated with task switching can be reliably detected (Allport, Styles, \& Hsieh, 1994). In typical paradigms that assess cognitive flexibility, participants perform on one of two or more different tasks on identical stimuli in each experimental trial, with a task cue indicating the relevant task. Usually, reaction times (RTs) and error rates are elevated after a switch from one task to another, as compared with subsequent performance on the same task. These task-switching costs that index an individual's cognitive flexibility vary considerably between individuals (Miyake et al., 2000) and are highly heritable with heritability estimates of $81 \%$, as indicated by twin studies (Friedman et al., 2008).

Much empirical work has been dedicated to explain why task switching elicits behavioral costs. A central concept in the literature is the task set that refers to mental representations constraining behavior in the service of the ongoing task (Monsell, 2003). Even though the performance impairment on switch trials can be reduced by preparation time and predictability of the task switch, residual switch costs remain that are attributed to task-set reconfiguration (TSR). TSR describes the entirety of cognitive processes associated with the abandonment of the initial and the initiation of the subsequent task set. The importance of task-set reconfiguration can be best illustrated with patients with brain damage to the frontal lobes or the basal ganglia. Such lesions sometimes lead to perseverative behavior - that is, the patient sticks to a 
specific task and is not capable of aborting its execution when appropriate (Allison, 1966). This impairment to abandon a task set after its initiation is contrasted by utilization behavior that can be caused by lesions to the same structures. Utilization behavior is a profound deficit to refrain from using or acting on items and stimuli in the proximal environment, even in the clear absence of any relevance for current goals (Lhermitte, 1983). It can be regarded as a deficit to maintain a goal-relevant task set and shield it against sources of distraction. With regards to the complementary nature of these two phenomena, the term "stability-flexibility-paradox" has been coined (Mayr \& Keele, 2000): During task execution, cognitive processes need to be stabilized in order to avoid premature task termination due to distraction, but as soon as task execution is no longer appropriate, an immediate disengagement is indicated in favor of a different task. To resolve this paradox, an executive control mechanism has been proposed that inhibits the to-be-abandoned task set in order to facilitate the initiation of the second task. Evidence for this backward inhibition effect has been accumulated in a series of experiments (Mayr \& Keele, 2000): Participants performed three different choice reaction tasks and switched tasks on each trial. The third trials in two different possible sequences were compared: Trials in which the participants switched back to a recently abandoned task set (sequence A-B-A) or to a less recently abandoned task set (sequence $\mathrm{A}-\mathrm{B}-\mathrm{C}$ ). According to the backward inhibition account, switching from task-set A to B goes along with an active inhibition of task-set A. As the inhibition decays slowly over time, reactivating a recently abandoned task set requires overcoming the inhibition. This leads to an additional processing demand on the third trial of the $\mathrm{A}-\mathrm{B}-\mathrm{A}$ sequence as compared with the $\mathrm{A}-\mathrm{B}-\mathrm{C}$ sequence that is reected in elevated RTs. The backward inhibition effect in task switching has been replicated in various experiments and is not attributable to stimulus-driven negative priming but is tied to top-down control and can partially account for residual switch costs - that is, task-set reconfiguration. An interesting property of the backward inhibition effect is that it is a double-edged sword: It is thought to accelerate task switching by reducing proactive interference from a no-longerrelevant task set. On the down side, however, this inhibition has to be overcome once a previously inhibited task set becomes relevant again, which leads to performance impairments in terms of prolonged response times.

In recent years, task-switching paradigms have been brought to the neuroscientific laboratory to assess the biological correlates of cognitive flexibility. In line with the aforementioned role of the frontal lobe in perseverative and utilization behavior, Rogers et al. (1998) reported performance deficits during task switching in patients with damage to the left frontal cortex. Within the frontal lobe, the inferior frontal junction (IFJ), a structure in the vicinity of the junction of the inferior frontal and the inferior precentral sulci, seems to be of major importance for task switching. A meta-analysis of 16 studies using fMRI showed consistently higher activation in the IFJ during switch than during repeat conditions (Derrfuss, Brass, Neumann, \& von Cramon, 2005). A further important neural structure for task switching is the dorsolateral prefrontal cortex (DLPFC). MacDonald, Cohen, Stenger, and Carter (2000) found increased activation of the DLPFC in preparation of task execution thatwas attributed to the active maintenance of task-related representations in working memory - a memory system with a wellknown implication for task switching (Baddeley, Chincotta, \& Adlam, 2001). The frontal and prefrontal cortices do not work in isolation. Functional circuits between striatal and prefrontal areas have been described (Alexander, DeLong, \& Strick, 1986), and neurocomputational models of executive control assume that the striatum has a gating function and contributes to goal-directed behavior by sending go- and nogo signals to the PFC, indicating whether working-memory representations need to be updated or maintained (Hazy, Frank \& O'Reilly, 2007). This designated role of striatal regions in cognitive flexibility and stability suggests that this structure contributes to task switching. And indeed, various studies provide evidence for a role of the striatum in task switching. Gu et al. (2008) found higher activity in the caudate nucleus on switch trials than on repeat trials. In a study by Cools, Sheridan, Jacobs and D'Esposito (2007), the flexible switching between stimulus categories in a workingmemory task activated the striatum during encoding. Recently, Stelzel, Basten, Montag, Reuter, and Fiebach (2010) provided evidence for the joint effort of the frontal cortex and striatal regions for task-switching performance. Using functional connectivity analysis, they were able to demonstrate a stronger coupling of the caudate nucleus and the IFJ during task switching. The striatum and the prefrontal cortex are the target areas of the mesocortical dopaminergic pathway that originate in the midbrain's ventral tegmental area (VTA) and the substantia nigra. Early evidence that dopaminergic neurotransmission may affect task switching stems from Rogers et al. (1998). They reported that patients suffering from Parkinson's disease who have abnormalities in their dopaminergic system due to a progredient loss of dopaminergic neurons in the substantia nigra show decreased task-switching performance as compared with healthy controls. Pharmacological treatment with L-dopa, however, can remediate this deficit, which points toward a causal role of dopamine in task switching (Cools, Barker, Sahakian, \& Robbins, 2003). Consistently, performance measures drop in healthy participants when treated with dopamine $\mathrm{d} 2$ receptor antagonists (Mehta, Manes, Magnol, Sahakian, \& Robbins, 2004). Given the high heritability (Friedman et al., 2008) and the strong individual variation in task switching (Miyake 
et al., 2000), a molecular genetic approach that compares carriers of different allelic variants affecting dopaminergic neurotransmission is a feasible complimentary strategy to the work in patients with abnormalities to their dopaminergic system and to the pharmacological challenge studies. A recent genetic imaging study compared performance and neural activation patterns in carriers of different allelic variants of the dopamine $\mathrm{d} 2$ receptor gene DRD2 (Stelzel et al., 2010). Dopamine $\mathrm{d} 2$ receptors are most prominently expressed in the striatum. The single nucleotide polymorphism (SNP) rs1800497 (DRD2/ANKK1 Taq Ia) was shown to determine the density of these receptors in vivo by means of positron-emission tomography: Carriers of at least one A1 allele $(\mathrm{A} 1+)$ have a $30 \%$ reduced striatal receptor density as compared with carriers of the homozygous A2/A2 (A1-) variant (Ritchie \& Noble, 2003). Stelzel et al. (2010) reported significantly reduced task-switching costs in carriers of the A1+ variant. This performance difference was paralleled by an increase of the blood oxygen level dependent (BOLD) response in the IFJ in A1-carriers. In this group, the BOLD signal correlated positively with the behavioral switch costs, and the dorsal striatum showed a stronger functional connectivity with the IFJ on switch trials. Furthermore, it was shown that the density of gray matter in the midbrain is reduced in carriers of the $\mathrm{A} 1+$ genotype (Cerasa et al., 2009; Montag, Weber, Jentgens, Elger, \& Reuter, 2010). The finding that genetic variants associated with a reduced DRD2 density benefit task switching performance seems to be at odds with the pharmacological evidence that DRD2 antagonists increase task-switching costs. This apparent contradiction can be resolved when considering the distinction between tonic and phasic dopaminergic activity in the striatum and its relationship to cognitive exibility (Bilder, Volavka, Lachman, \& Grace, 2004): Spontaneous activity of VTA neurons leads to a low yet constant level of extracellular dopamine. VTA neurons respond to behaviorally salient stimuli with burst firing, which triggers high levels of dopamine release on top of the tonic dopamine. This phasic release leads to extracellular dopamine concentrations that are high enough to stimulate postsynaptic DRD2. In response, striatal neurons send updating signals to the PFC, which helps to establish new representation in working memory. In contrast with phasic dopamine, tonically released dopamine does not bind to postsynaptic receptors but to presynaptic autoreceptors, which attenuates the phasic dopamine release and the updating signals. It is conceivable that the reduced receptor density associated with the A1+ variant pertains to mostly presynaptic receptors (Zhang et al., 2007). Thus, genetically determined, reduced presynaptic receptor density reduces the inhibitory inuence of tonic dopamine on the phasic transmitter release. This is backed up by the finding that, indeed, $\mathrm{A} 1+$ carriers have an increased dopaminergic synthesis capacity in the striatum (Laakso et al., 2005). The higher levels of phasic dopamine lead to stronger updating signals that may contribute to a higher degree of flexibility during task switching. DRD2 antagonists, in contrast, block both pre- and postsynaptic receptors and abolish DRD2-mediated updating signals at all, leading to a decrement in performance. Until now, only the biological correlates of global task- switching performance have been investigated. Especially on the molecular genetic level, little is known about the subprocesses that might be the main target of the genetic pathway and, as such, mediate the association between a given gene and a phenotype. Task-switching costs are mainly attributed to cognitive processes involved in the reconfiguration of task sets, which is, amongst others, the inhibition of the to-be-abandoned task set. In the present study, we sought to investigate whether genetically determined variations in dopamine $\mathrm{d} 2$ receptor density can account for individual differences in the magnitude of the backward inhibition effect. It was proposed that backward inhibition works by lateral inhibition - that is, by inhibiting irrelevant information in the moment working memory contents need to be updated with new information (Mayr \& Keele, 2000). Thus, there is reason to assume that $\mathrm{d} 2$ receptor-mediated dopaminergic neurotransmission affects the inhibition of tobe-abandoned task sets. To clarify this issue, we used the previously described odd-man-out task because it provides an elegant way to isolate the cognitive process associated with the inhibition of irrelevant task-sets. Stelzel et al. (2010) showed reduced task-switching costs in DRD2 A1+ carriers in a traditional task-switching paradigm. Because a stronger backward inhibition is thought to relate to better taskswitching performance (Mayr \& Keele, 2000), we expect the backward inhibition to be stronger in $\mathrm{A} 1+$ carriers, in case we find an association between the polymorphism and the phenotype. Besides the DRD2 Taq Ia polymorphism, other genetic variations in the dopaminergic system have been related to behavioral phenotypes in the domain of executive control. On the DRD2 gene itself, at least two more polymorphisms (rs6277 and rs2283265) are possible candidates for an association in the present study's scope (Markett, Montag, \& Reuter, 2010). Furthermore, the val158met polymorphism on the gene coding for the dopamine degrading enzyme Catechol-O-Methyltransferase (COMT) seems to affect inhibitory control processes as well, either alone (Egan et al., 2001) or in interaction with variants on the DRD2 gene (Reuter et al., 2005). To achieve a more thorough understanding of the molecular genetic underpinnings of inhibitory control, we also seek to explore a possible influence of these three genetic variants on the backward inhibition effect. Note, however, that the main focus of the present research lies on the role of the DRD2 Taq Ia polymorphism. Therefore, the results of the remaining polymorphisms are presented in a supplement. 


\section{Method}

Participants

A total of $N=100$ (males $n=16$, females $n=84$; age $M=23: 25, S D=5: 13$ ) Caucasian participants took part in the experiment. Participants were free of neurological or psychiatric disorders as assessed by a short screening questionnaire. Twenty-four participants reported to be regular smokers, and two males stated that they consumed more than five alcoholic beverages a week. However, any influence of acute alcohol intake on performance during the experiment could be ruled out. Participants were recruited from the Bonn Gene Brain Behavior Project's data bank and had previously delivered DNA samples together with their informed written consent to be contacted for further behavioral testing.

\section{Task}

The behavioral protocol was a variant of the odd-man-out task as described in Mayr and Keele (2000), Experiment 1B. Stimulus presentation was controlled by Eprime 2.0 (www. pstnet.com). Figure 1 depicts some consecutive trials in the course of the experiment. Throughout the experiment, a white frame was visible in the middle of the otherwise black screen. Each trial started with the presentation of a white letter in the middle of the frame, which served as a cue and indicated the relevant task for the following stimulus display. The stimulus display consisted of four blue rectangles that deviated from the others in one out of three different dimensions: Rectangles deviant on the motion dimension showed oscillatory movements either in the vertical or in the horizontal plane (random selection of movement direction). Rectangles that deviated on the orientation dimension were tilted either $45^{\circ}$ clockwise or counter-clockwise from the upright standing position (random selection of tilting direction). Deviation on the size dimension was accomplished by a $50 \%$ increase or decrease in size (again, random selection). The verbal Cue (letters B, O, G for the German words Bewegung [motion], Orientierung [orientation], or Größe [size]) was visible throughout stimulus presentation in the middle of the frame. Participants were instructed to identify the rectangle deviant on the cued dimension and then indicate its position by a button press to a spatially corresponding key on a computer keyboard. The stimulus display terminated upon a button press and was replaced by a stimulus-cue interval (black screen with empty white frame). Timing of stimulus presentation was the same as in the original Experiment 1B by Mayr and Keele. The cue was presented for $100 \mathrm{~ms}$ and the stimulus display until a response was delivered. The stimulus-cue interval lasted for $100 \mathrm{~ms}$. Timing parameters were chosen to maximize the backward inhibition effect in order to allow for an appropriate range of individual variation. Including instructions and breaks, the experiment took about $15 \mathrm{~min}$. The order of the three tasks (orientation, motion, size) was pseudorandomized for each participant so that no task cue was repeated in consecutive trials. This resulted in only two possible trial types: lag-2 and lag-3 repetitions.

\section{Genotyping}

DNA was extracted from buccal cells. Automated purification of genomic DNA was conducted by means of the MagNA Pure RLC system using a commercial extraction kit (MagNA Pure LC DNA isolation kit; Roche Diagnostics, Mannheim, Germany). Genotyping was performed by means of a realtime polymerase chain reaction (RT-PCR) using melting curve detection analysis on a Light Cycler System (Roche Diagnostics, Mannheim, Germany). The protocols (TIB MOLBIOL, Berlin, Germany) for the RT-PCR were as follows:

Forward primer: 5' -CGGCTGGCCAAGTTGTCTAA -3' Backward primer: 5'- AGCACCTTCCTGAGTGTCATCA -3'

Anchor hybridisation probe: 5'- LCRed640TGAGGATGGCTGTGTTGCCCTT-phosphate-3'

Sensor hybridisation probe: 5'- CTGCCTCGACCAGCACT - fluorescein -3 '

For details on the genotyping of COMT and the other two DRD2 polymorphisms, please see the supplementary material.

Procedure

After obtaining their informed-written consent, participants were led to a testing booth where they were given eight training trials with feedback. Then, participants completed 200 trials in two blocks without feedback. Participants were debriefed afterward. The study was carried out in accordance with the ethical principles of the Declaration of Helsinki of the World Medical Association and was approved by the ethics committee of the German Psychologist Association.

\section{Results}

The genotype frequency for the DRD2/ANKK1 Taq Ia polymorphism was as follows: $\mathrm{A} 1 / \mathrm{A} 1, n=6 ; \mathrm{A} 1 / \mathrm{A} 2, n=$ 29; A2/A2, $n=65$ (test for Hardy-Weinberg equilibrium, $\left.\chi^{2}(1)=.17, \mathrm{~ns}\right)$. As is common in the literature, participants were grouped according to the A1 allele into an $\mathrm{A} 1+(\mathrm{A} 1 / \mathrm{A} 1$ and $\mathrm{A} 1 / \mathrm{A} 2)$ and an $\mathrm{A} 1-(\mathrm{A} 2 / \mathrm{A} 2)$ group because the homozygous A1/A1 genotpye is quite seldom found in Caucasian populations. Before analyzing individual differences depending on the A1 allele, we computed $t$ tests for dependent measurements to replicate the backward inhibition effect in 
Fig. 1 Exemplary trial sequence from the experiment: Depicted is the experiment's microstructure (task cue, task, task-cue interval) and macrostructure (lag2 repetitions and non repetitions). Please note that the cue letters in the actual experiment were German while the figure shows the English translation. The white arrows illustrate the rectangles' movement and were not shown on screen

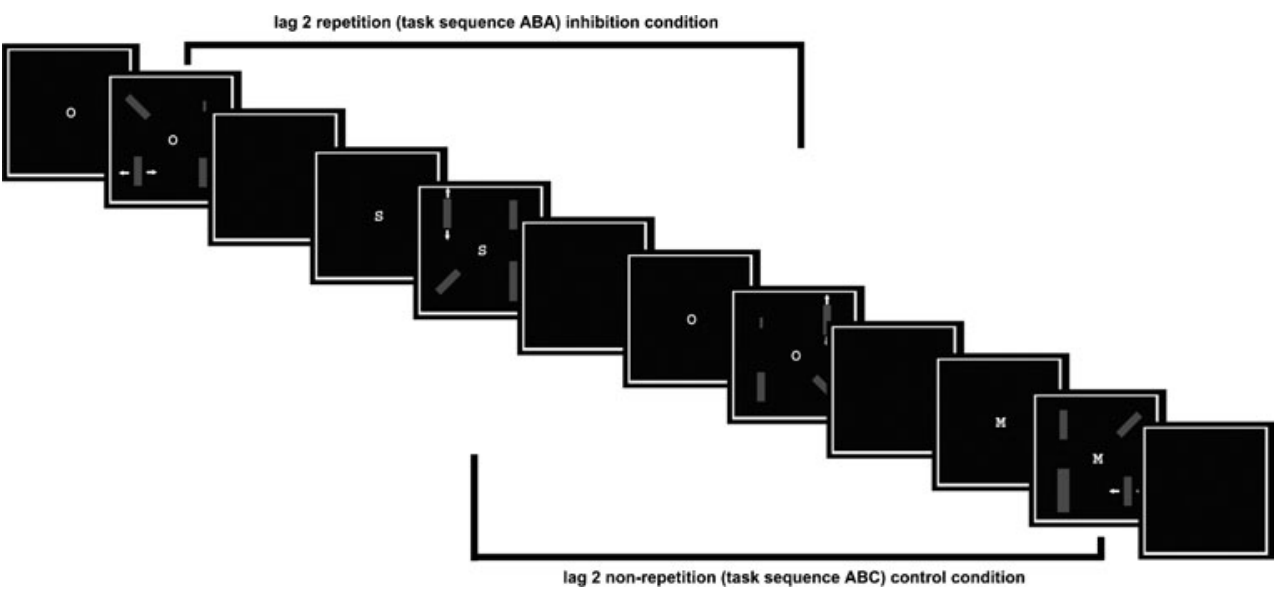

our entire sample. Overall performance was very high $(95 \%$ correct trials on average); nonetheless, only RTs from correct trials were analyzed. Figure 2 shows the RT data in the backward inhibition (task sequence $\mathrm{ABA}$ ) and in the control condition (ABC). As was expected, RTs were elevated when participants switched back to a recently abandoned task set as compared with a switch to a third task, $t(99)=-8.196$, $p<.001$, Cohen's $d=.825$. For the error rates, however, no difference between the two conditions was detectable (backward inhibition, $M=0.056, S D=0.08$; control $M=0.052$, $S D=0.08), t(99)=-1.616, p=.11$, Cohen's $d=.16$. In order to analyze gene effects on RTs, an ANOVA for repeated measurements was calculated with task condition (inhibition vs. control) as within- and DRD2 genotype (A1+ vs. A1-) as between-subjects factors. Means and standard errors, depending on task condition and DRD2 genotype, are depicted in Fig. 3. The within- subjects effect turned out to be significant,

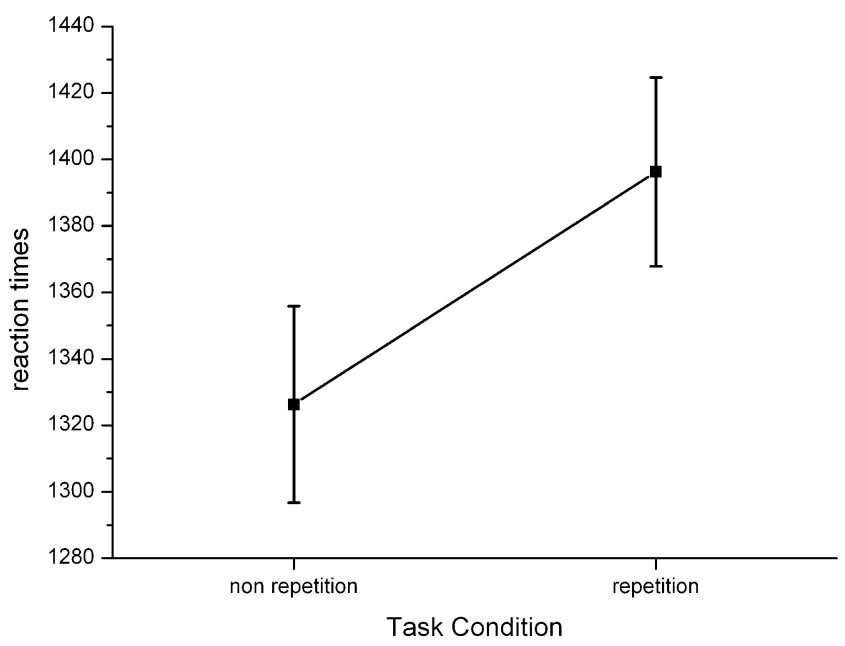

Fig. 2 Mean reaction times and SEMs from the within subject analysis: Reaction times are significantly elevated on lag-2 repetitions compared to lag-2 non-repetitions
$F(1,98)=76.618, p<.001, \eta^{2}=.439$, which reflects the RT increase associated with the backward inhibition. Most importantly, the two-way interaction was significant, $F(1,98)=6.321, p=.014, \eta^{2}=.061$. Carriers of the $\mathrm{A} 1+$ genotype showed a backward inhibition effect that was almost twice as large as that for A1- carriers (98.5 \pm 16.4 vs. $54.57 \pm 9.3)$. There was no main effect for the DRD2 gene, $F(1,98)=0.084 ; p=.772$. We computed a similar ANOVA model for the error rates. No significant effects could be detected (all $p \mathrm{~s}<.10$ ).

\section{Discussion}

In the present study, we provided evidence that genetically determined differences in the dopaminergic system infuence the backward inhibition effect during task switching. Carriers of the A1+ variant of the DRD2 rs1800497 polymorphism who have a $30 \%$ decreased density of dopamine $\mathrm{d} 2$ receptors in the striatum showed a stronger backward inhibition effect, which was about twice as large as that of carriers of the homozygous A2/A2 (A1-) variant. The data fit into the results by Stelzel et al. (2010), who reported higher flexibility in terms of reduced task-switching costs in $\mathrm{A} 1+$ carriers. Bringing the results together, we speculate that a stronger inhibition of irrelevant task sets facilitates the establishment of a new task set and thus a quicker execution of the new task in $\mathrm{A} 1+$ carriers. The designated role of the striatum in cognitive control is a gatekeeper function that informs the prefrontal cortex to hold representations either stable and shielded against distraction or to update working memory contents with new contextual information (Hazy et al., 2007; McNab \& Klingberg, 2008). The previous results by Stelzel et al. (2010) did not allow for a conclusion whether a low density of $\mathrm{d} 2$ receptors enhances cognitive flexibility by shielding active task sets less efficiently and thus placing fewer demands on task-set reconfiguration or by supporting 
more efficient updating signals. Our present results point toward a more efficient updating mechanism that operates at least in part by inhibiting no-longer-relevant representations.

The idea that the active inhibition or directed ignoring of irrelevant or no longer relevant information is a key executive control mechanism (Zacks \& Hasher, 1994) has a long tradition in cognitive psychology and can account for many empirical phenomena in the literature such as inhibition of return (Posner \& Cohen, 1984), negative priming (Tipper, 2001) and retrieval-induced forgetting (Anderson, Bjork, \& Bjork, 1994). The investigation of the biological correlates of these inhibitory functions, however, appears to be still in its infancy. Related yet distinct phenotypes have been studied using neuroimaging and molecular genetics. One example is response inhibition, as assessed by the stop-signal paradigm (Logan \& Cowan, 1984). In this paradigm, participants are instructed to make speeded responses in a choice reaction task but to withhold their responses when a no-go signal occurs briefly after task onset. Performance on these stop-signal trials depends on the participant's ability to inhibit response delivery after the motor program has been initiated. Various studies have confirmed that these stop-signal trials activate a network comprising various prefrontal sites, including dorsolateral and orbital prefrontal cortex and the inferior frontal cortex and parts of the basal ganglia (Aron \& Poldrack, 2006).

A first genetic imaging study has addressed the molecular genetic foundations of response inhibition (Congdon, Constable, Lesch, \& Canli, 2009). Stop-signal trials activated the inferior prefrontal cortex and the basal ganglia's subthalamic nucleus more strongly in carriers of genetic variants

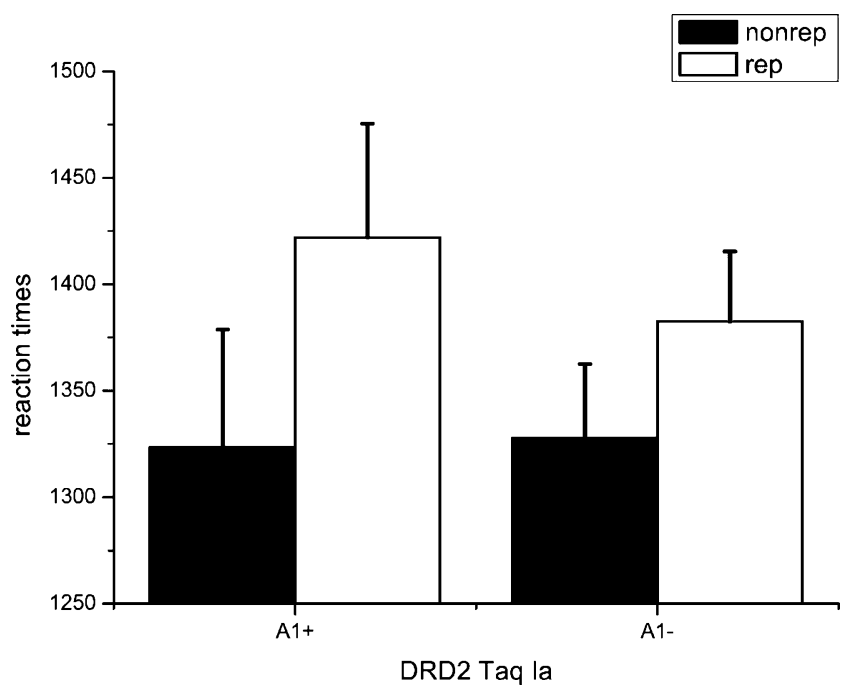

Fig. 3 Mean reaction times and SEMs from the between subject analysis. The backward inhibition effect is increased in carriers of the $\mathrm{A} 1+$ genetic variant (left panel) compared to homozygous A2/A2 (A1-) carriers (right panel) associated with reduced dopamine degradation capacity in the prefrontal cortex (catechol-O-methyltransferase COMT 158 met carriers) and in the basal ganglia (dopamine transporter SLC6A3 9-allele carriers), respectively. Another means to study inhibitory control is the antisaccade task. In this paradigm, participants are required to inhibit reflexive eye movements to stimuli appearing in their peripheral visual field and to execute a saccade in the opposite direction. Neuroimaging studies have shown that succesful antisaccade performance activates a fronto-parietosubcortical network comprising the DLPFC, the frontal and supplementary eye fields, the intraparietal sulcus, and the thalamus (for review, see Hutton \& Ettinger, 2006). Ettinger et al. (2008) combined eyetracking, functional imaging, and molecular genetics and found that carriers of the COMT val158 variant showed reduced activity in the ventro and dorsomedial prefrontal cortex during antisaccade performance. A third paradigm related to task-set switching is the Wisconsin Card Sorting Test (WCST), a clinical assessment tool for the diagnosis of prefrontal deficits. In this test, participants are required to learn stimulus-response mappings and to subsequently adapt to new mappings by trial-and-error learning. The abandonment of a stimulus-response mapping after the mapping principle is no longer rewarded requires similar inhibitory control as does task-set switching. Imaging work has shown that the WCST elicits activation in the dorsolateral and ventrolateral prefrontal cortex and the caudate nucleus, and there is evidence that the activity in the dorsolateral prefrontal cortex is inuenced by the COMT gene (Egan et al., 2001). Konishi et al. (1999) compared the activation pattern between the WCST and the stop-signal task. Although both tasks activated different networks, conjoint activity was found in the right inferior frontal cortex, which was interpreted as an inhibition node in both networks. Even though task-set switching shows a considerable overlap with performance in the stop-signal task, the antisaccade paradigm, and the WCST regarding the required cognitive operations, they still assess different endophenotypes of cognitive flexibility (Robbins, 2007). Antisaccade and stop-signal performance depends on the ability to suppress an automatic and otherwise appropriate motor response, whereas WCST and task-switching performance require an attentional shift from one stimulus dimension to another (i.e., an extradimensional shift) and a simultaneous inhibition of the tendency to apply rules associated with a no-longer-relevant stimulus dimension. The extradimensional shift in the WCST is furthermore based on a flexible behavioral adjustment in the face of omitted reward, whereas the shift in task-set switching paradigms is solely based on external instruction. Miyake et al. (2000) have shown that at least three different components of executive functioning are dissociable. Interestingly, shifting and inhibition seem to be two different control mechanisms. The disentanglement of these functions' dynamic interplay 
during task performance in various paradigms is a probing question for future research. At present, the picture of inhibitory control as a mechanism of executive control is only about to emerge. It is still unclear whether inhibitory control is a unitary phenomenon that operates across different domains or whether different tasks require different inhibitory mechanisms. Molecular genetics, as applied in the present study, is a further level of analysis besides behavioral assessment and neuroimaging and provides a feasible tool for the dissociation of different executive control processes. Moreover, the genetic approach gives - in contrast with fMRI studies - direct evidence for the neurochemical pathways involved in executive control. As a starting point, the aforementioned evidence indicates that genetic variations associated with dopamine degradation seem to underlie inhibitory control in the stop-signal paradigm, the antisaccade task, and the WCST, whereas in our present data set, a genetic variation related to the dopamine $\mathrm{d} 2$ receptor affects inhibitory control in task-set switching. Importantly, the COMT val158met polymorphism did not account for individual differences in inhibitory control in our present data set (see supplementary material). Although this result questions the assumption of unitarity of inhibitory control across different tasks, a firm conclusion is not possible because we did not apply other measures of inhibitory control simultaneously. Future work should therefore address this issue more thoroughly and aim at a dissociation of various genetic influences on the different conceptualizations of inhibitory control in the same data set to see whether all three genes relate to the same executive control network or whether different mechanisms can already be disentangled at the molecular genetic level. To the best of our knowledge, this is the first study that has examined the genetic basis of a more fine-grained endophenotype of cognitive exibility within the task-set switching paradigm. So far, genetic association studies have rather focused on more global phenotypes. In another strain of research that is comparable to our study but with another focus, Stelzel, Basten, Montag, Reuter, and Fiebach (2009) have taken a closer look at the influence of genetic variants on working memory and have found that the frequently reported association of the COMT and the DRD2 genes with global working memory performance is mainly driven by an effect on the updating component of working memory. We believe that this is a promising approach for future research that will help to go from correlation to explanation and, eventually, to gain an in depth understanding of the molecular genetics of cognition.

Author Note We are grateful to our students Anna-Lena Salz, Bernd Lachmann, Daniela Halm, Jennifer Hillebrand, Niklas Gilsdorf, and Stefanie Hackstein for valuable discussions and their help in data acquisition.

\section{References}

Alexander, G. E., DeLong, M. R., \& Strick, P. L. (1986). Parallel organization of functionally segregated circuits linking basal ganglia and cortex. Annual Reviews Neuroscience, 9, 357-381.

Allison, R. S. (1966). Perseveration as a sign of difuse and focal brain damage. British Medical Journal, 2, 1027-1032.

Allport, D. A., Styles, E. A., \& Hsieh, S. (1994). Shifting intentional set: Exploring the dynamic control of tasks. In C. Umilta \& M. Moscovitch (Eds.), Attention and Performance XV (pp. 421-452). Hillsdale, NJ: Erlbaum.

Anderson, M. C., Bjork, R. A., \& Bjork, E. L. (1994). Remembering can cause forgetting: Retrieval dynamics in long-term memory. Journal of Experimental Psychology: Learning, Memory, and Cognition, 20, 1063-1087.

Aron, A. R., \& Poldrack, R. A. (2006). Cortical and subcortical contributions to stop signal response inhibition: Role of the subthalamic nucleus. Journal of Neuroscience, 26, 2424-2433.

Baddeley, A., Chincotta, D., \& Adlam, A. (2001). Working memoy and the control of action: Evidence from task-switching. Journal of Experimental Psychology: General, 130, 641-657.

Bilder, R. M., Volavka, J., Lachman, H. M., \& Grace, A. A. (2004). The catechol-O-methyltransferase polymorphism: Relations to the tonic-phasic dopamine hypothesis and neuropsychiatric phenotypes. Neuropsychopharmacology, 29, 1943-1961.

Cerasa, A., Gioia, M. C., Tarantino, P., Labate, A., Arabia, G., Annesi, G., et al. (2009). The DRD2 TaqIa polymorphism associated with changed midbrain volumes in healthy individuals. Genes, Brain and Behavior, 8, 459-463.

Congdon, E., Constable, R. T., Lesch, K. P., \& Canli, T. (2009). Influence of SLC6A3 and COMT variation on neural activation during response inhibition. Biological Psychology, 81, 144-152.

Cools, R., Barker, R. A., Sahakian, B. J., \& Robbins, T. W. (2003). Ldopa medication remediates cognitive inflexibility, but increases impulsivity in patients with parkinson's disease. Neuropsychologia, 41, 1431-1441.

Cools, R., Sheridan, M., Jacobs, E., \& D'Esposito, M. (2007). Impulsive personality predicts dopamine-dependent changes in frontostriatal activity during component processes of working memory. Journal of Neuroscience, 27, 5506-5514.

Derrfuss, J., Brass, M., Neumann, J., \& von Cramon, D. Y. (2005). Involvement of the inferior frontal junction in cognitive control: Meta-analyses of switching and stroop studies. Human Brain Mapping, 25, 22-34.

Egan, M. F., Goldberg, T. E., Kolachana, B. S., Callicott, J. H., Mazzanti, C. M., Straub, R. E., et al. (2001). Effect of comt val108/158 met genotype on frontal lobe function and risk for schizophrenia. Proceedings of the National Academy of Sciences, 98, 6917-6922.

Ettinger, U., Kumari, V., Collier, D. A., Powell, J., Luzi, S., Michel, T. M., et al. (2008). Catechol-O-methyltransferase (COMT) val158met genotype is associated with bold response as a function of task characteristic. Neuropsychopharmacology, 33, 3046-3057.

Friedman, N. P., Miyake, A., Young, S. E., Defries, J. C., Corley, R. P., \& Hewitt, J. K. (2008). Individual differences in executive functions are almost entirely genetic in origin. Journal of Experimental Psychology: General, 137, 201-225.

Gu, B.-M., Park, J.-Y., Kang, D.-H., Lee, S. J., Yoo, S. Y., Jo, H. J., et al. (2008). Neural correlates of cognitive inexibility during taskswitching in obsessive-compulsive disorder. Brain, 131, 155-164.

Hazy, T. E., Frank, M. J., \& O'Reilly, R. C. (2007). Towards an executive without a homunculus: Computational models of the prefrontal cortex/basal ganglia system. Philosophical Transaction of the Royal Society London B: Biological Sciences, 362, 16011613. 
Hutton, S. B., \& Ettinger, U. (2006). The antisaccade task as a research tool in psychopathology: A critical review. Psychophysiology, 43, 302-313.

Konishi, S., Nakajima, K., Uchida, I., Kikyo, H., Kameyama, M., \& Miyashita, Y. (1999). Common inhibitory mechanism in human inferior prefrontal cortex revealed by event-related functional mri. Brain, 122, 981-991.

Laakso, A., Pohjalainen, T., Bergman, J., Kajander, J., Haaparanta, M., Solin, O., et al. (2005). The al allele of the human d2 dopamine receptor gene is associated with increased activity of striatal l-amino acid decarboxylase in healthy subjects. Pharmacogenetic Genomics, 15, 387-391.

Lhermitte, F. (1983). "Utilization behaviour" and its relation to lesions of the frontal lobes. Brain, 106, 237-255.

Logan, G., \& Cowan, W. (1984). On the ability to inhibit thought and action: A theory of an act of control. Psychological Review, 91, 295397.

MacDonald, A. W., Cohen, J. D., Stenger, V. A., \& Carter, C. S. (2000). Dissociating the role of the dorsolateral prefrontal and anterior cingulate cortex in cognitive control. Science, 288, 1835-1838.

Markett, S. A., Montag, C., \& Reuter, M. (2010). The association between dopamine DRD2 polymorphisms and working memory capacity is modulated by a functional polymorphism on the nicotinic receptor gene chrna4. Journal of Cognitive Neuroscience, 22, 1944-1954.

Mayr, U., \& Keele, S. W. (2000). Changing internal constraints on action: The role of backward inhibition. Journal of Experimental Psychology: General, 129, 4-26.

McNab, F., \& Klingberg, T. (2008). Prefrontal cortex and basal ganglia control access to working memory. Nature Neuroscience, 11, 103-107.

Mehta, M. A., Manes, F. F., Magnol, G., Sahakian, B. J., \& Robbins, T. W. (2004). Impaired set-shifting and dissociable effects on tests of spatial working memory following the dopamine $\mathrm{d} 2$ receptor antagonist sulpiride in human volunteers. Psychopharmacology, 176, 331-342.

Miyake, A., Friedman, N. P., Emerson, M. J., Witzki, A. H., Howerter, A., \& Wager, T. D. (2000). The unity and diversity of executive functions and their contributions to complex "frontal lobe" tasks: A latent variable analysis. Cognitive Psychology, 41, 49-100.

Monsell, S. (2003). Task switching. Trends in Cognitive Sciences, 7 , $134-140$
Montag, C., Weber, B., Jentgens, E., Elger, C., \& Reuter, M. (2010). An epistasis effect of functional variants on the BDNF and DRD2 genes modulates gray matter volume of the anterior cingulate cortex in healthy humans. Neuropsychologia, 48, 1016-1021.

Posner, M. I., \& Cohen, Y. (1984). Components of visual orienting. In H. Bouma \& D. Bouwhuis (Eds.), Attention and Performance $X$ (pp. 531-556). Hillsdale, NJ: Erlbaum.

Reuter, M., Peters, K., Schroeter, K., Koebke, W., Lenardon, D., Bloch, B., et al. (2005). The influence of the dopaminergic system on cognitive functioning: A molecular genetic approach. Behavioral Brain Research, 164, 93-99.

Ritchie, T., \& Noble, E. P. (2003). Association of seven polymorphisms of the $\mathrm{d} 2$ dopamine receptor gene with brain receptor-binding characteristics. Neurochemical Research, 28, 73-82.

Robbins, T. W. (2007). Shifting and stopping: Fronto-striatal substrates, neurochemical modulation and clinical implications. Philosophical Transactions of the Royal Society of London B: Biological Sciences, 362, 917-932.

Rogers, R. D., Sahakian, B. J., Hodges, J. R., Polkey, C. E., Kennard, C., \& Robbins, T. W. (1998). Dissociating executive mechanisms of task control following frontal lobe damage and parkinson's disease. Brain, 121, 815-842.

Stelzel, C., Basten, U., Montag, C., Reuter, M., \& Fiebach, C. J. (2009). Effects of dopamine-related gene-gene interactions on working memory component processes. European Journal of Neuroscience, 29, 1056-1063.

Stelzel, C., Basten, U., Montag, C., Reuter, M., \& Fiebach, C. J. (2010). Frontostriatal involvement in task switching depends on genetic differences in $\mathrm{d} 2$ receptor density. Journal of Neuroscience, 30, 14205-14212.

Tipper, S. P. (2001). Does negative priming reect inhibitory mechanisms? A review and integration of conicting views. Quarterly Journal of Experimental Psychology, 54, 321-343.

Zacks, R. T., \& Hasher, L. (1994). Directed ignoring: Inhibitory regulation of working memory. In D. Dragenbach \& T. H. Carr (Eds.), Inhibitory processes in attention, memory, and language (pp. 214-264). San Diego, CA: Academic Press.

Zhang, Y., Bertolino, A., Fazio, L., Blasi, G., Rampino, A., Romano, R., et al. (2007). Polymorphisms in human dopamine $\mathrm{d} 2$ receptor gene affect gene expression, splicing, and neuronal activity during working memory. Proceedings of the National Acadademy of Sciences, 104, 20552-20557. 